\title{
Effect of High-speed Solenoid Valve Dynamic Characteristics on Downstream Transient Spray Atomization of Bipropellant Thruster
}

\author{
Zhen Zhang ${ }^{* 1,2}$, Ping Wang ${ }^{2}$, Fengshan Wang ${ }^{2}$, Yu Hu², Yusong $\mathrm{Yu}^{1}$, Qi Chen ${ }^{1}$ \\ ${ }^{1}$ Beijing Jiaotong University, Beijing, China \\ ${ }^{2}$ Beijing Institute of Control Engineering, Beijing, China. \\ *Corresponding author email: 3203zhen@163.com
}

\begin{abstract}
The new generation of intelligent satellites is now in urgent demand for bipropellant thrusters in minimum impulse mode without limitation. Thruster's impulse performance involving combustion efficiency and impulse repeatability strongly correlates to transient spray atomization. Accordingly, the transient spray atomization under impulse working condition is investigated to optimize thruster dynamic response. In order for thrusters to work reliably in short-impulse mode and to further improve its impulse repeatability, the solenoid valve must be improved firstly to achieve high-speed switch with stable flow supply. In the simulation using OpenFOAM, the differences in switch speed, valve stroke and throttle layout are all investigated in comparison and optimization. The fluctuating flowrate provided from the solenoid outlet can be further used as boundary conditions to study downstream swirl injector atomization. Based on the interFoam solver and 2D wedge physical model, spray simulations of MMH (monomethyl hydrazine) and NTO (nitrogen tetroxide) propellants under fluctuating condition are conducted. When the upstream flowrate stabilizes faster, the atomization stability can also be enhanced, thereby improving the impulse repeatability of thrusters in combustion. Atomization is rather unstable when upstream flowrate fluctuates violently, but this fluctuation can enhance the primary breakup of droplets. Compared with MMH spray, NTO spray from outer swirl injector is more sensitive to different upstream fluctuation. Throttle layout plays the most significant roles in transient spray development. In the short-impulse firing test, the thruster with improved high-speed solenoid valve can just work under the impulse time of $4 \mathrm{~ms}$ with poor repeatability. But this repeatability can be improved with the increase of impulse time, and satisfy the requirement at impulse command of $8 \mathrm{~ms}$. This experimental result made a good agreement with the simulation, thus showing that only when transient atomization of MMH spray and NTO spray both develop into the steady state in 5ms after valve opening, the impulse performance is able to be reliably achieved.
\end{abstract}

\section{Keywords:}

High-speed solenoid valve; Swirl injector; Transient spray atomization; Bipropellant thruster.

\section{Introduction}

The bipropellant thruster is a low-thrust liquid rocket engine used to stabilize or change the attitude of spacecrafts. It usually works in variable impulse modes and uses solenoid valve to control its switching. The minimum impulse width is only a few milliseconds and the start-up times are up to millions of times. The smaller the thruster impulse is, the higher the control precision of the spacecraft can be. Therefore, with the development of new generation satellites, the requirement of autonomous and intelligent control demands thrusters are able to work in an unrestricted mode. That is, thrusters are needed to offer minimum impulses with high stability and repeatability in an instant, less than $10 \mathrm{~ms}$. It is beneficial to improve the attitude control efficiency of the satellite, reduce switching times and save the consumption of propellant in orbit ${ }^{[1]}$. 
Injector spray plays a primary determining role in bipropellant thruster for spacecraft propulsion system. The injector is designed to balance the homogeneous mixing of propellant and liquid film cooling by organizing spray atomization. Under the effect of different valve characteristics, whether two propellants can achieve better atomization and mixing in milliseconds is a key to improve impulse performance.

Traditionally, the combustion efficiency and cooling performance often need to be validated and calibrated through firing-test runs at a high cost. But spray simulation analysis indicates a large amount of data and information, aiming for failure prevention and design optimization. Many researchers have been experimentally and numerically conducting work to study swirl spray ${ }^{[2-4]}$. In this study, we firstly discuss the effect of high-speed valve opening: switch speed, valve stroke and different throttle layouts. We conducted numerical simulations employing the VOF method to simulate the flowrate fluctuation from the valve outlet, and compared these results in different cases. Then the flowrate fluctuation with swirling effect was taken as new inlet boundary of a pair of swirl coaxial injectors to analysis the atomization development of $\mathrm{MMH}$ and NTO propellants in milliseconds.

In the development of magnetic devices such as power inductors, the effective permeability and induction of the devices is changed by the method of pre-biased magnetic field ${ }^{[5]}$. Under the effect of the bias magnetic field of the permanent magnet, the coil current of the solenoid valve raises significantly faster when powering up. Thus, the electromagnetic force increases rapidly to switch the armature. When power is cut, the current rapidly reduced to close the valve seat. The current curve was used to testify the movement of armature in valve, as shown in Figure 1 (left). As coil voltage is applied, current goes up gradually under inductive effect. When armature starts to move, a back potential causes the first knee in current curve, then it backs up with an inflection point as the armature move end. The same can be said in return process. Simulation analysis in Ansoft Maxwell ${ }^{6]}$ shows that the open time with pre-biased magnetic field is around $3 \mathrm{~ms}$, only $30 \%$ of the normal solenoid valve under the same condition of voltage supply. In the test, the open time of high-speed valve is only $2.9 \mathrm{~ms}$ as shown at the first inflection point of current curve in Figure 1 (right). This indicates that it is possible for thrusters to work at any impulse command of more than $3 \mathrm{~ms}$. However, whether steady combustion can be constructed depends on the spray development after valve opening.
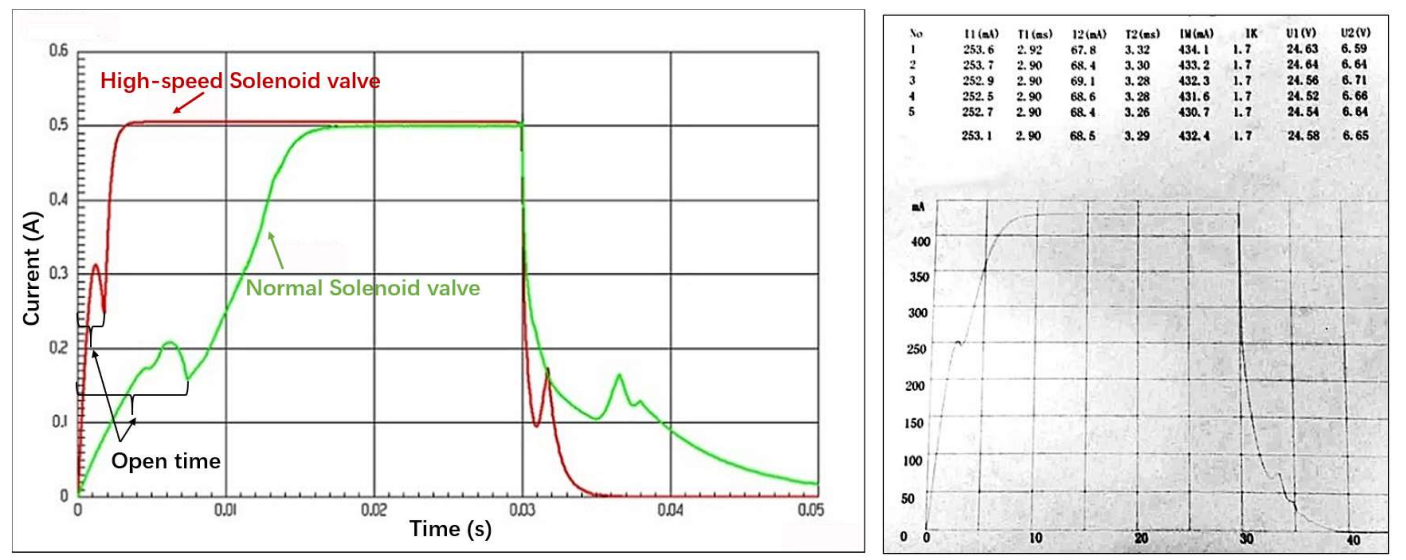

Figure 1. Simulation result (left) and test data (right) of high-speed solenoid valve with around $3 \mathrm{~ms}$ switching time In the process of valve opening, the flow is inevitably unstable, and the throttle orifice plate which is set in the flow passage to control equivalence ratio generates cavitation, thus causing fluctuation. The transient spray from swirl injector downstream is significantly determined by the fluctuating flow. The dynamic mesh and the interFOAM solver are used to analyse this fluctuation in the following $5 \mathrm{~ms}$ after valve opening under different conditions. 


\section{Methods}

\section{(1) Simulation Solver}

The inner flow in valve switching and spray atomization from swirl injector downstream are simulated using the VOF (volume of fluid) method. Deshpande et al. published a detailed description of the interFoam-code in OpenFOAM, and showed its capabilities in interface capturing ${ }^{[7][8]}$. For a low-thrust bipropellant thruster, the diameter of injector is very small with low-velocity spray. That is, the Reynolds number is under 2000 , and there is no turbulent model used in the following simulations.

Simulations are conducted in two sections. The first is to investigate the fluctuating flow rate in the valve switching process using dynamic mesh. And then the fluctuating flowrate in these cases can be provided as inlet boundary condition for swirl injector spray in the second section. For 2D simulation, swirling effect can be provided in swirlFlowRatelnletVelocity condition with RPM (revolutions per minute) value matching its transient flowrate.

\section{(2) Physical model, boundary condition, and fluid properties assumptions}

In this simulation study, the focus is on comparisons of jet breakup and spray development. Therefore, 2D simulation cases are conducted to describe the macroscopic shape and stabilization of the entire spray field, comparing spray visualization and dimensionless total surface area ratio in different cases ${ }^{[9]}$.

There are two kinds of axial-symmetric domain used for valve-switch flow and injector-spray simulations, respectively as shown in Figure 2 and Figure 3 . In these cases, the wedge mesh with 5 degree is used to simulate inner-flow in valve and swirl spray from injectors. The mesh in spray domain is refined as small as $10 \mu \mathrm{m} \times 10 \mu \mathrm{m}$. It has proved adequate to capture the breakup droplets with an average diameter of above 50 $\mu \mathrm{m}$ measured by PDPA (phase doppler particle analyzer).

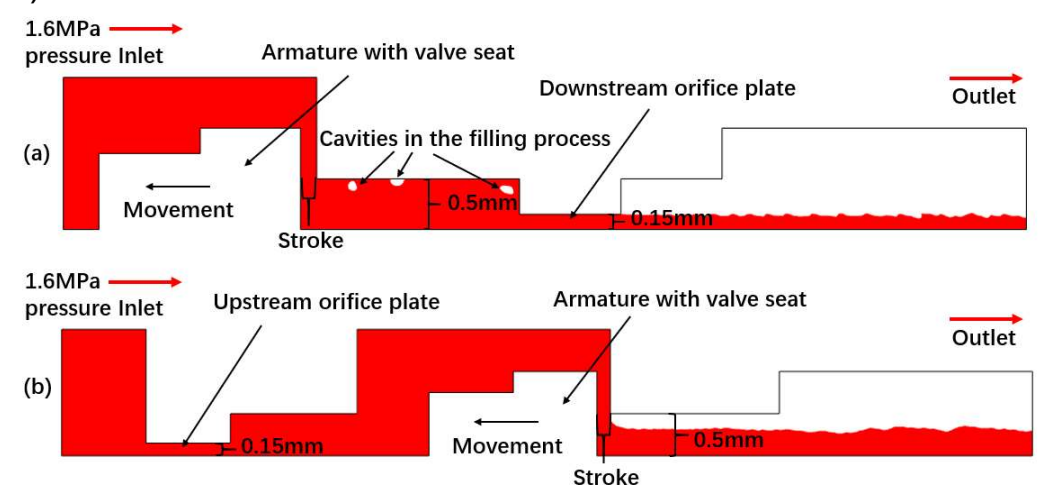

Figure 2. Simulation domains of valve simulation with downstream orifice plate(a) and upstream orifice plate(b)

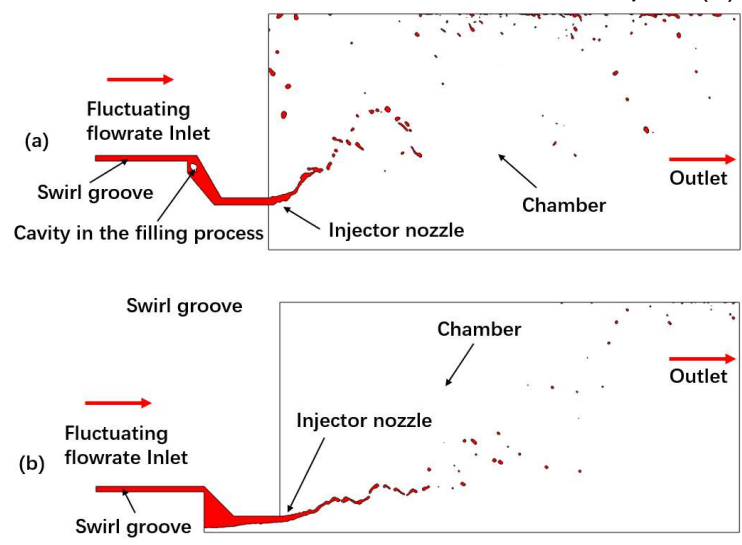

Figure 3. Simulation domains of outer injector spray for NTO (a) and inner injector spray for MMH (b) 
All conditions are listed in Table 1, with different propellants, switch speeds, valve strokes and throttle layouts.

Table 1. Conditions

\begin{tabular}{ccccc}
\hline $\begin{array}{c}\text { Propellant } \\
\text { properties }\end{array}$ & $\begin{array}{c}\text { Ambient } \\
\text { pressure }(\mathrm{Pa})\end{array}$ & $\begin{array}{c}\text { Valve switch } \\
\text { Speed }(\mathrm{m} / \mathrm{s})\end{array}$ & $\begin{array}{c}\text { Valve stroke } \\
(\mathrm{mm})\end{array}$ & $\begin{array}{c}\text { Throttle } \\
\text { layout }\end{array}$ \\
\hline MMH, NTO & 100 & $0.125,0.25$, & $0.15,0.25$ & Upstream, \\
& & $0.5,1$ & & downstream \\
\hline
\end{tabular}

\section{(3) Validation}

To validate the simulation method and physics model, the current method was compared with experimental result in the steady-state spray. For this comparison study, both these two inlet conditions are constant flowrate input, showing a good agreement in the conical liquid film with umbrella-shape, similar spray angle, breakup length and droplet size, as shown in Figure 4. The comparison indicates that the simulation setup is suitable for atomization analysis using VOF methodology.

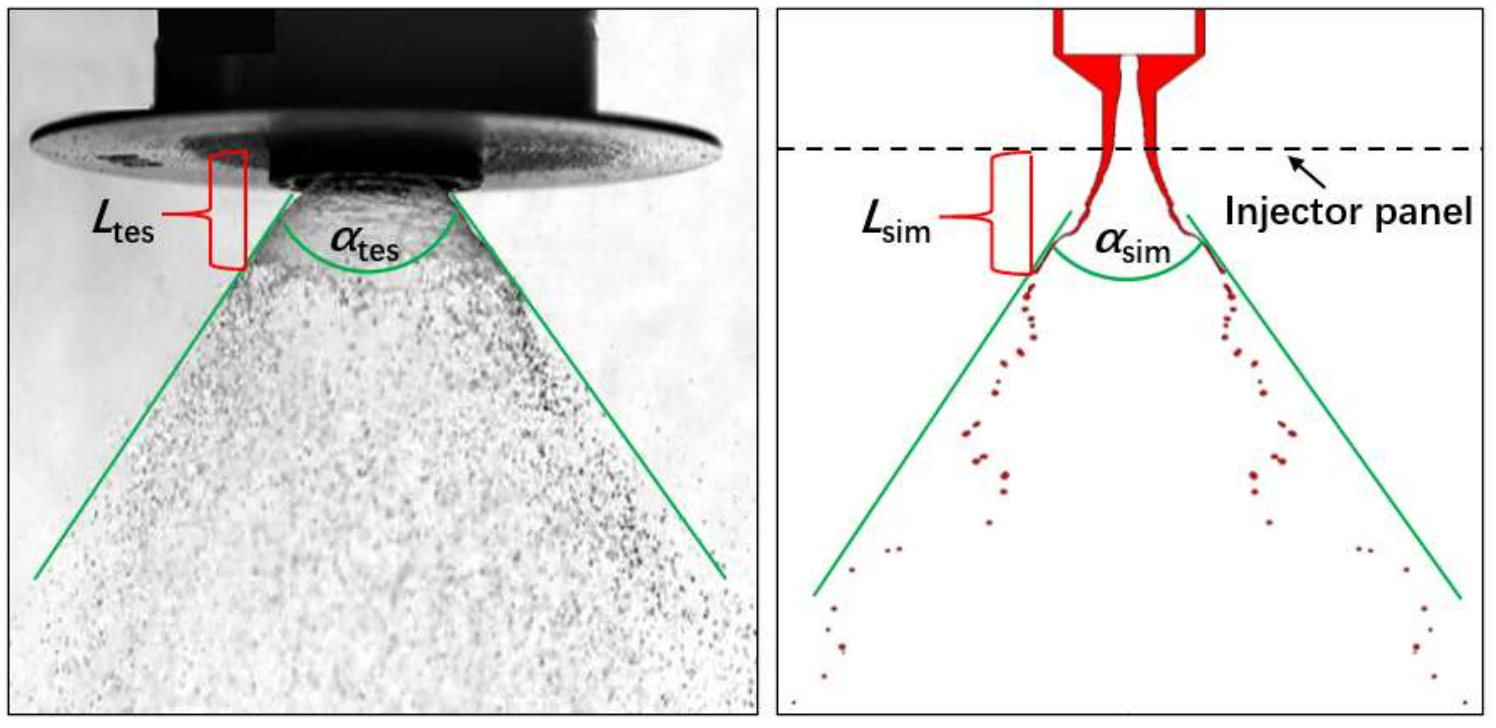

Figure 4. Comparison of validation between experimental image (left) and simulative result (right) by high-speed camera with umbrella-shape, similar spray angle ( $\alpha_{\text {tes }}$ and $\left.\alpha_{\text {sim }}\right)$ and breakup length ( $L_{\text {tes }}$ and $\left.L_{\text {sim }}\right)$.

\section{Results and Discussion}

The effects of different conditions on transient spray breakup are compared and discussed in this section. The investigated effects are switch speed, valve stroke and throttle layout for propellants of MMH and NTO, respectively. Under the impulse command of $8 \mathrm{~ms}$ On time, we focused on the spray process in the following $5 \mathrm{~ms}$, considering the $3 \mathrm{~ms}$ delay of solenoid valve switch as mentioned above.

\section{(1) Effect of propellant properties}

NTO is oxidizer with low surface tension and viscosity, compared with MMH as fuel. Therefore, in centrifugal thrusters, NTO is often designed in outer swirls due to its more easily atomized properties.

Figure 5 shows differences in flowrate fluctuation between $\mathrm{MMH}$ and NTO. In the switching process, throttle orifice plate inevitably causes cavitation in the flow. That is the reason why fluctuation in NTO flow with lower surface tension is more significant. After flowing out from the valve outlet, these two propellants will enter two different separate nozzles. NTO flows into the outer annular nozzle and $\mathrm{MMH}$ flows into the inner circular nozzle to form liquid-liquid coaxial centrifugal spray. 


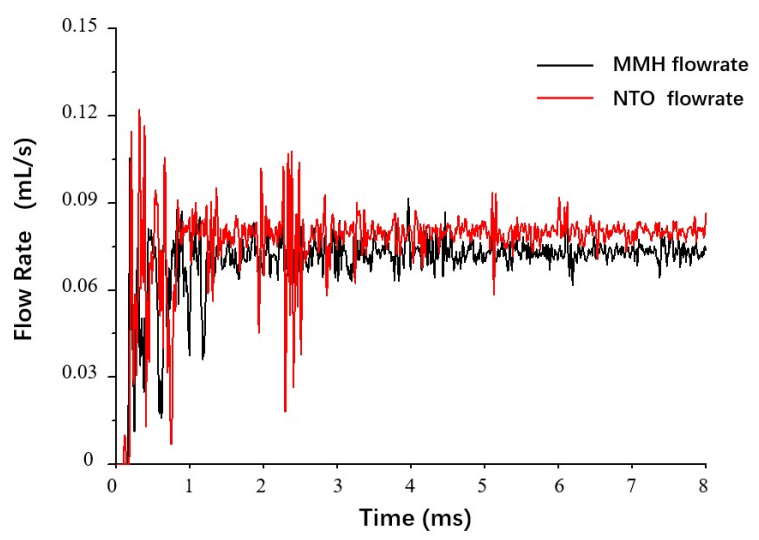

Figure 5. Comparison of flowrate fluctuation between $\mathrm{MMH}$ and NTO with downstream orifice plate at $0.5 \mathrm{~m} / \mathrm{s}$ switch speed and $0.25 \mathrm{~mm}$ stroke.

At the beginning of injection, the swirl jet forms a conical liquid film under the action of centrifugal force after leaving the nozzle. The front of the liquid film interacts with ambient shearing gas, so that the conical film forms a thin umbrella-shaped structure. K-H instability wave is generated on the surface of liquid film due to ambient gas disturbance and boundary layer effect. What needs to be emphasized is that there is more obvious surface fluctuation just from the nozzle exit than spray with constant flow. When the surface wave develops to a certain extent with higher amplitude over the film thickness, the liquid film begins to breakup and breakup into ligaments and droplets. After the first atomization, the liquid ligaments and droplets still move with a high velocity, and continue to split under the aerodynamic interaction, resulting in secondary atomization ${ }^{[10]}$. The typical development of swirl spray under the condition of inlet fluctuation is shown in Figure 6.

In the spray process, MMH flow in the inner injector can fill inside with many cavities under fluctuation, and gradually develops from an unstable state to a stabler state with a hollow vortex core after $2 \mathrm{~ms}^{[11]}$. Because the center is occupied by the inner nozzle, NTO liquid film is directly formed and ejected without hollow vortex core, after the filling process with cavitation. The spray angle of NTO spray is bigger, and secondary breakup, rebound and splash can be formed after impingement on the chamber wall.

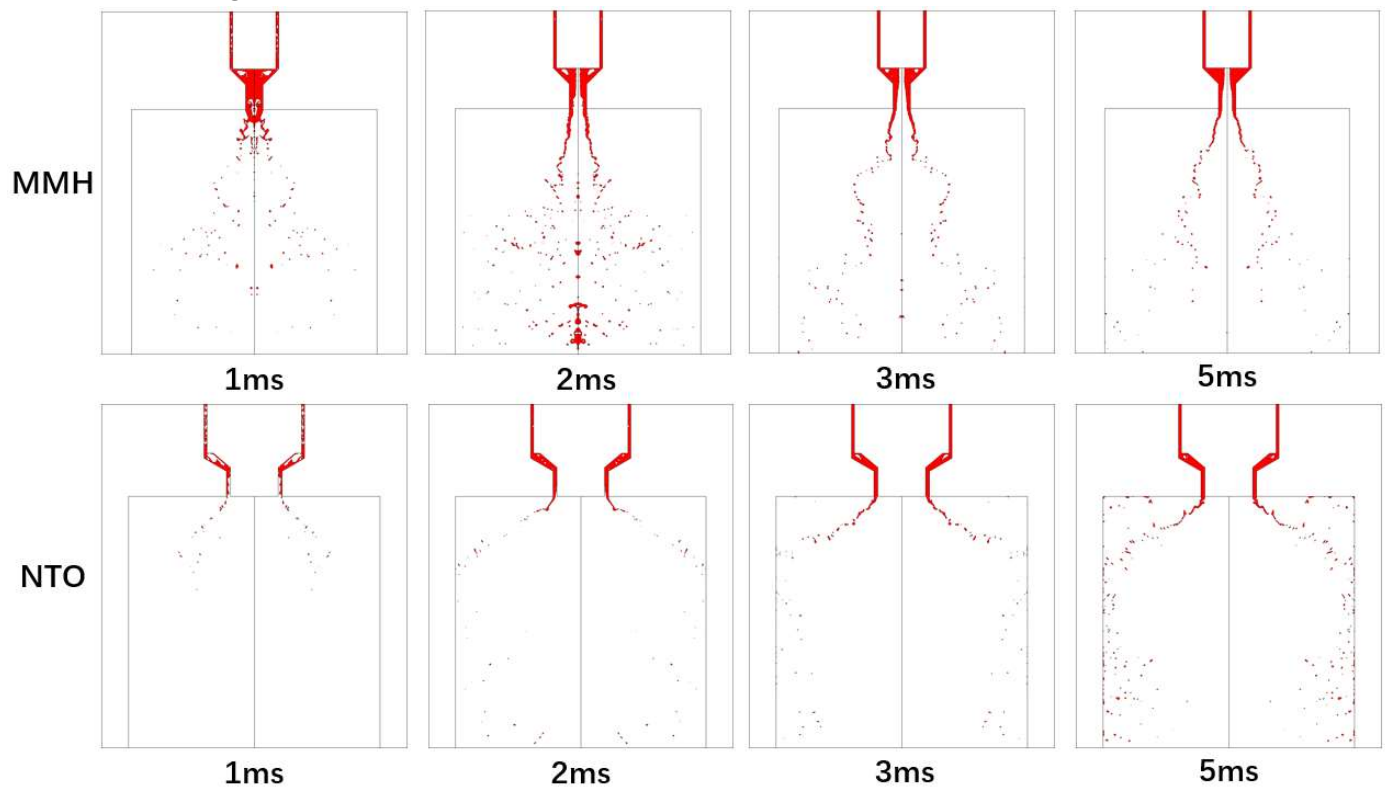

Figure 6. The development of MMH and NTO transient spray atomization over time after valve opening. (2) Effect of switch speed 
Different switch speed of armature affects the fluctuation from valve outlet. As switch speed decreases, the $\mathrm{MMH}$ flow tends to become more unstable, as shown in Figure 7 . While switch speed is $0.25 \mathrm{~m} / \mathrm{s}$, the fluctuation amplitude is only strong within first $2 \mathrm{~ms}$. When switch speed drops down to $0.125 \mathrm{~m} / \mathrm{s}$, the fluctuation amplitude of the flow surges significantly. By contrast, NTO flow is more sensitive to the effect of armature speed in opening process. When switch speed is reduced to $0.25 \mathrm{~m} / \mathrm{s}$, it is already sufficient to reach the threshold that causes violent fluctuation.

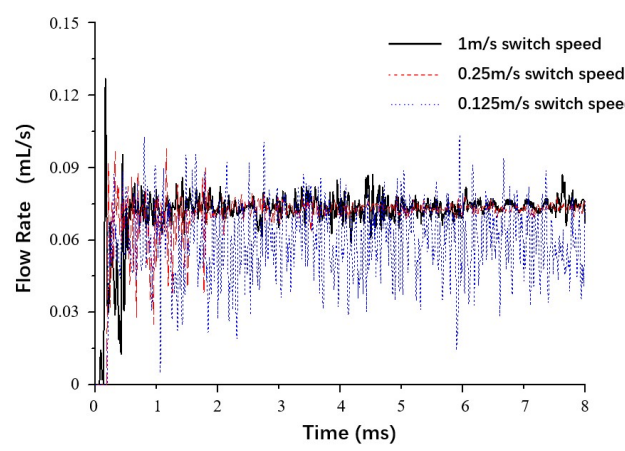

(a) $\mathrm{MMH}$

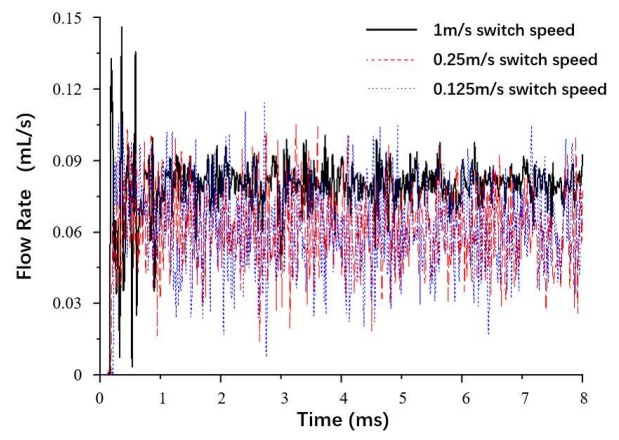

(b) NTO

Figure 7. Comparison of MMH and NTO flowrate fluctuation under different switch speed with downstream orifice plate and $0.15 \mathrm{~mm}$ stroke.

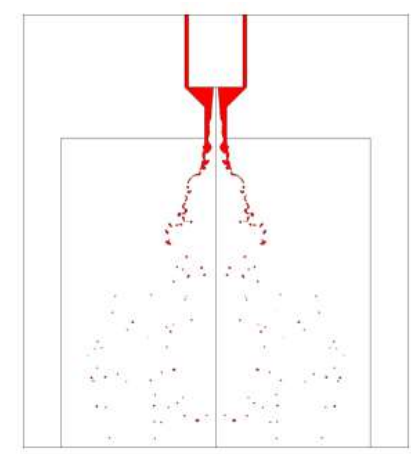

(a)

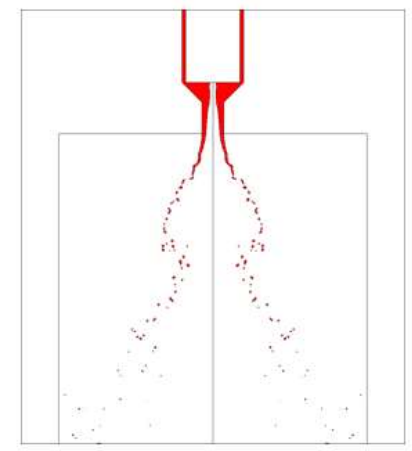

(b)

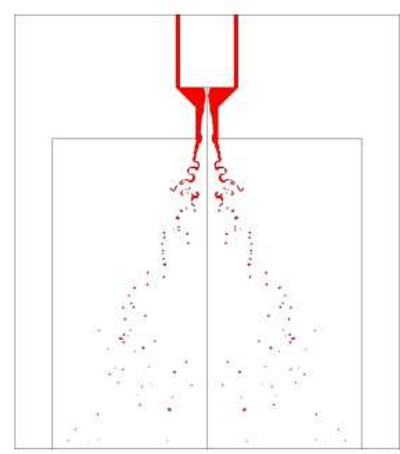

(c)

Figure 8. Comparison of MMH spray development at $5 \mathrm{~ms}$ under different switch speed: (a) $0.125 \mathrm{~m} / \mathrm{s}$ (b) $0.25 \mathrm{~m} / \mathrm{s}$ (c) $1 \mathrm{~m} / \mathrm{s}$, with downstream orifice plate and $0.15 \mathrm{~mm}$ stroke.

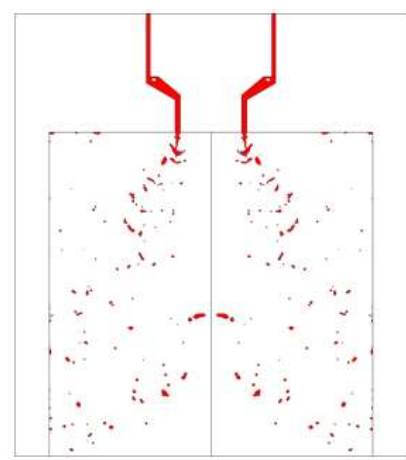

(a)

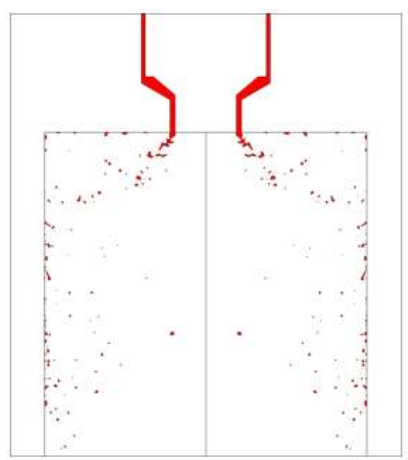

(b)

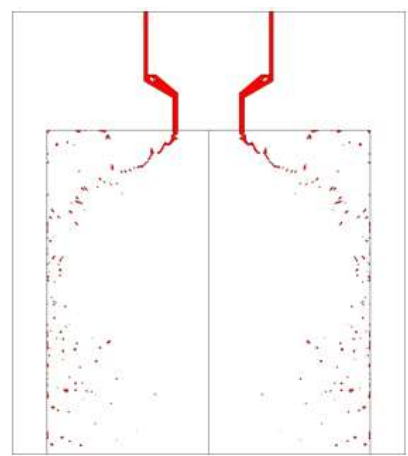

(c)

Figure 9. Comparison of NTO spray development at $5 \mathrm{~ms}$ under different switch speed: (a) $0.125 \mathrm{~m} / \mathrm{s}$ (b) $0.25 \mathrm{~m} / \mathrm{s}$ (c) $1 \mathrm{~m} / \mathrm{s}$, with downstream orifice plate and $0.15 \mathrm{~mm}$ stroke.

Figure 8 shows visual differences between spray flows at different switch speed from $\mathrm{MMH}$ inner injector and NTO outer injector at $5 \mathrm{~ms}$ after valve opening. For MMH spray, changes in ambient mean pressure seem to have slight effect on the spray atomization. When switch speed is low, violent fluctuation can significantly strengthen the disturbance, so as to obtain 
comparatively better droplets breakup, but its stability is worse than that of high-speed switch. Limited by the structural disadvantage of the outer nozzle, the spray atomization of NTO is relatively more difficult, even though the NTO property is conducive to atomization. Therefore, the atomization of NTO takes a relatively long time. In the instant time of just $5 \mathrm{~ms}$, the highspeed switch contributes to the intact and stable atomization as shown in Figure 9. However, when it works at low switch speed, strong upstream fluctuation compensates the breakup of liquid film and ligament.

\section{(3) Effect of valve stroke}

The valve stroke determines its opening gap, influencing cavitation generation. In Figure 11(a) and (b), for both MMH flow and NTO flow, increasing the stroke can greatly strengthen the flow stability with lower fluctuation. Even for the NTO flow case of low-speed switch with very violent fluctuation, the fluctuation can also get rapid attenuation and damping after increasing the stroke.

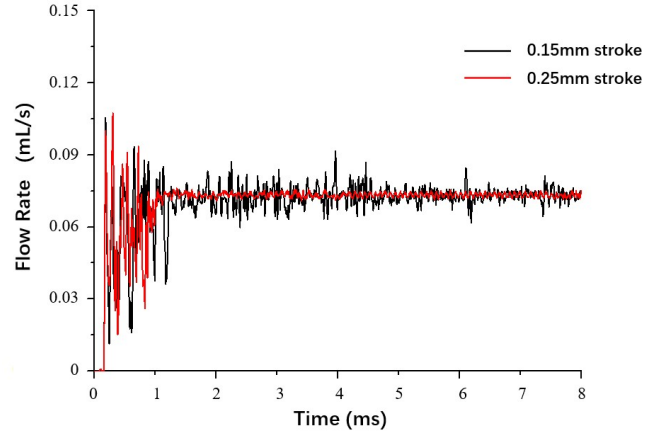

(a) $\mathrm{MMH}$

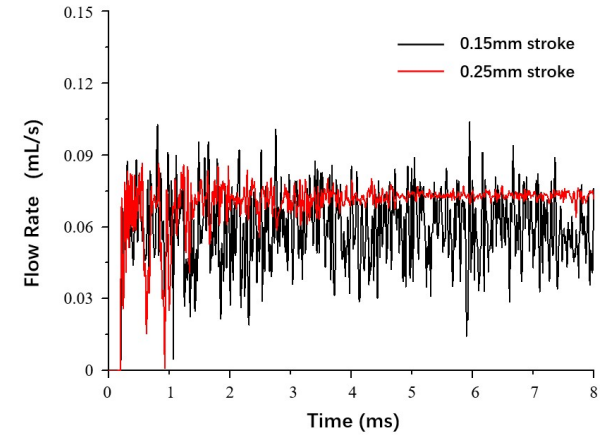

(b) NTO

Figure 11. Comparison of different valve strokes: (a) $\mathrm{MMH}$ flow at $0.5 \mathrm{~m} / \mathrm{s}$ switch speed with downstream orifice plate; (b) NTO flow, at $0.125 \mathrm{~m} / \mathrm{s}$ switch speed with downstream orifice plate

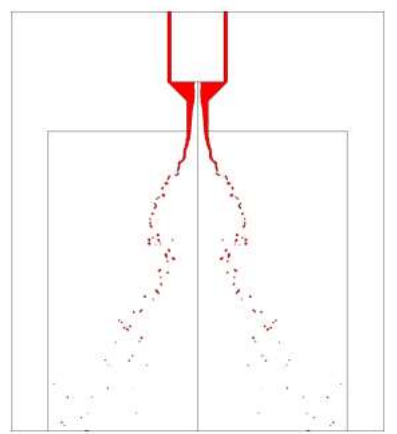

(a)

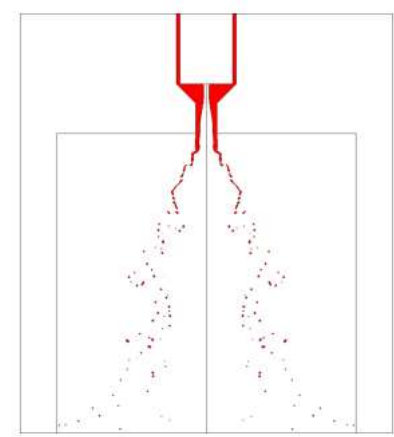

(b)

Figure 12. Comparison of MMH spray development at $5 \mathrm{~ms}$ under different valve strokes: (a) $0.15 \mathrm{~mm}$ (b) $0.25 \mathrm{~mm}$, at $0.25 \mathrm{~m} / \mathrm{s}$ switch speed with downstream orifice plate.

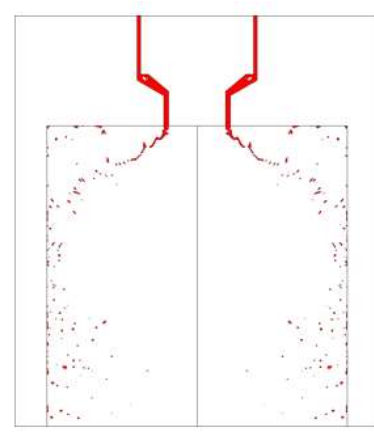

(a)

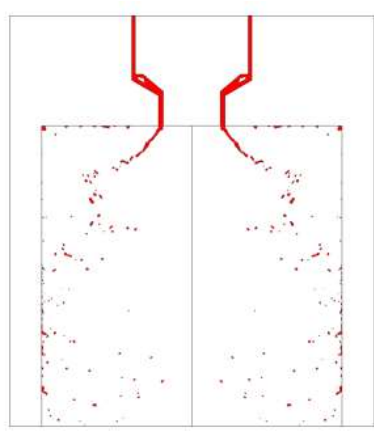

(b)

Figure 13. Comparison of NTO spray development at $5 \mathrm{~ms}$ under different valve strokes: (a) $0.15 \mathrm{~mm}$ (b) $0.25 \mathrm{~mm}$, at $1 \mathrm{~m} / \mathrm{s}$ switch speed with downstream orifice plate. 
Figure 12 and 13 demonstrate the visual differences between spray development at different strokes. In these comparative cases, the liquid film can form an umbrella-shaped structure with similar hollow vortex core, film breakup length and spray cone angle. The atomized droplets size of $\mathrm{MMH}$ tends to be smaller at larger valve stroke, while the atomized droplets of NTO is more dispersive in spatial distribution at larger valve stroke. These are more combustion-friendly characteristics for the following diffuse-burning process.

\section{(4) Effect of throttle layouts}

When the orifice plate is placed upstream of valve, the instantaneous flow characteristics are different from the layout of downstream throttle. Compared with the downstream throttle, upstream orifice plate layout leads to more stable but much lower transient flowrate as shown in Figure 14. Therefore, the swirl injection with lower velocity significantly weakens the aerodynamic interaction, which causes the decrease of atomization quality, especially for NTO spray with the structural deficiency in Figure 15.

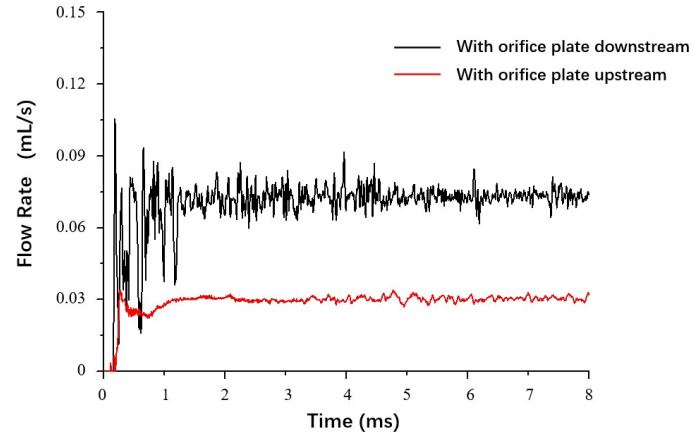

(a) $\mathrm{MMH}$

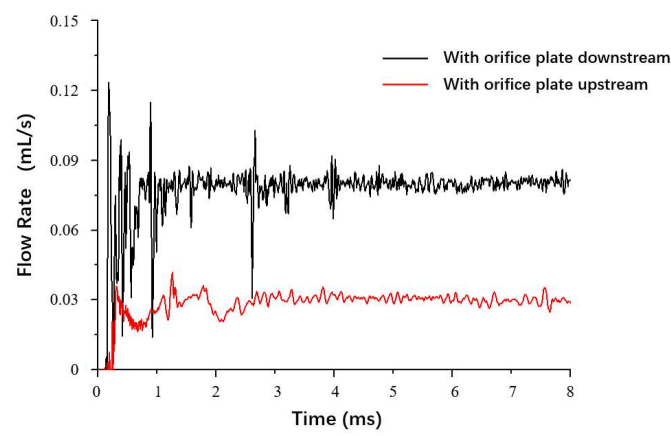

(b) NTO

Figure 14. Comparison of different throttle layouts: (a) MMH flow at $0.5 \mathrm{~m} / \mathrm{s}$ switch speed and $0.15 \mathrm{~mm}$ stroke; (b) NTO flow at $1 \mathrm{~m} / \mathrm{s}$ switch speed and $0.25 \mathrm{~mm}$ stroke.

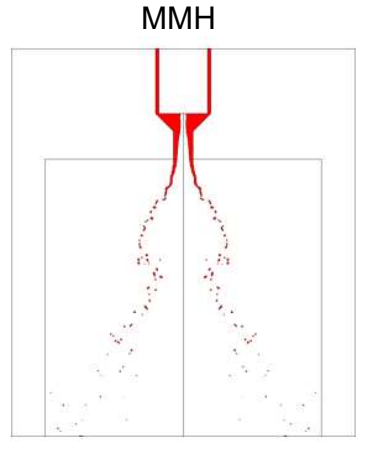

(a) downstream layout

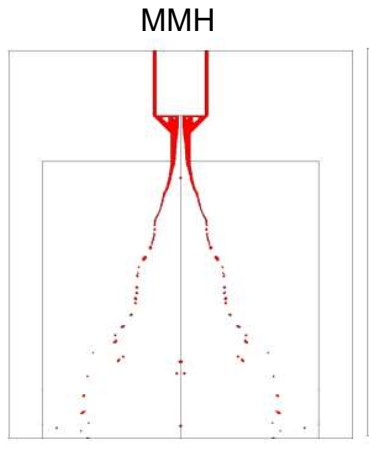

(b) upstream layout

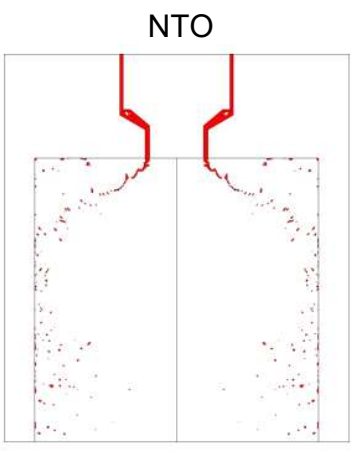

(c) downstream layout

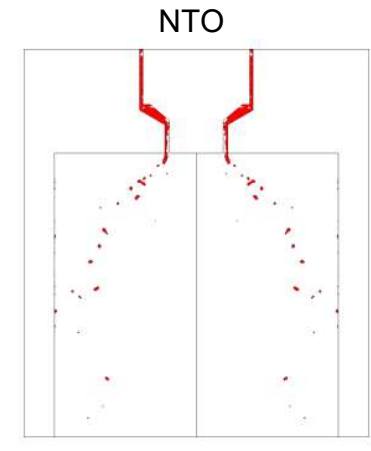

(d) upstream layout

Figure 15. Comparison of different throttle layouts: (a) downstream layout and (b) upstream layout with MMH flow at $0.5 \mathrm{~m} / \mathrm{s}$ switch speed and $0.15 \mathrm{~mm}$ stroke; (c) downstream layout and (d) upstream layout with NTO flow at $1 \mathrm{~m} / \mathrm{s}$ switch speed and $0.25 \mathrm{~mm}$ stroke.

In order to investigate the effect of above factors quantitatively, the total surface area of liquid flow is obtained by integrating all areas of the spray jet interface. And then the total surface area ratio in each case can be used for comparison by dividing the total surface area of constant flow. In Figure 16 (a), for MMH spray, the larger stroke and downstream throttle layout are more conducive to transient atomization, but the effect is not significant due to its structure. Low-speed switch seems to strengthen the breakup under strong fluctuation, but the resulting instability and inconsistency are negative for the following combustion. In Figure 16 (b), in the case of NTO spray, it is sensitive to the change in different throttle layouts. Using the downstream orifice layout can increase the surface area of spray by 2-3 times. Meanwhile, valve stroke and switch speed also have a certain effect on the spray development, but there 
is no unidirectional tendency. Regarding the above analysis, $1 \mathrm{~m} / \mathrm{s}$ switch speed, $0.15 \mathrm{~mm}$ valve stroke and downstream throttle layout should be a better combination for optimization.

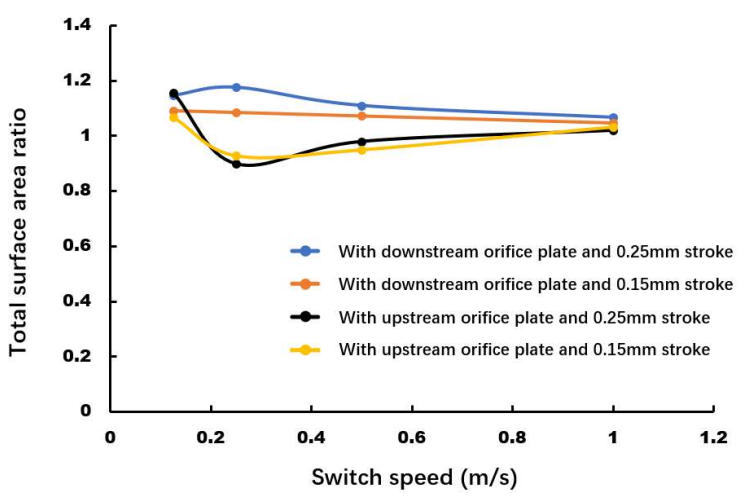

(a) $\mathrm{MMH}$

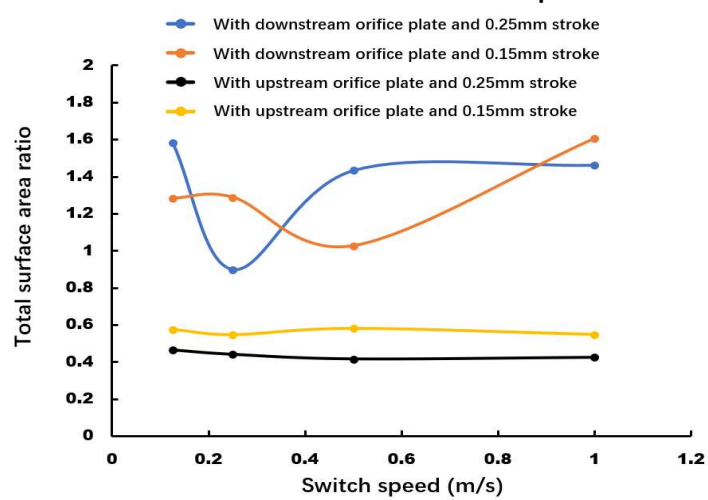

(b) NTO

Figure 16. Total Surface area ratio under different switch speed, valve stroke and throttle layouts.

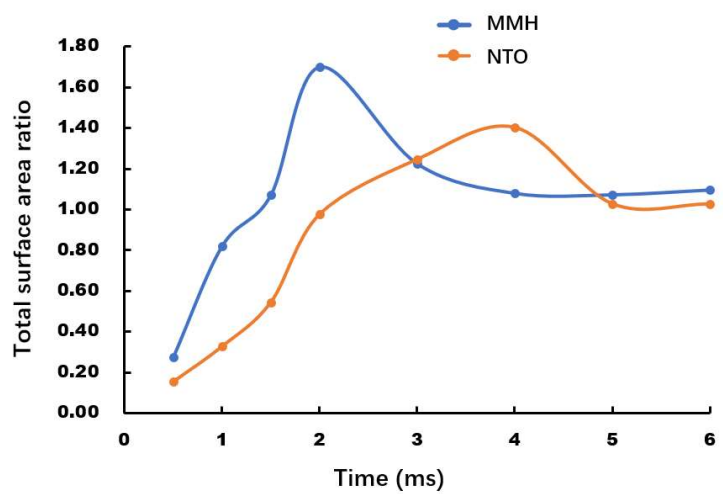

Figure 17. Total surface area ratio over time at $1 \mathrm{~m} / \mathrm{s}$ switch speed, $0.15 \mathrm{~mm}$ stroke, with downstream throttle layout Figure 17 reflects the dynamic process of transient atomization both in MMH spray and NTO spray with optimized option as described above. Due to the initial strong fluctuation of valve switch, the MMH spray reaches a peak at $2 \mathrm{~ms}$, which was $2 \mathrm{~ms}$ ahead of NTO spray. After that, the MMH spray gradually stabilizes after $3 \mathrm{~ms}$, while the NTO spray requires $5 \mathrm{~ms}$ back to around surface area ratio 1.
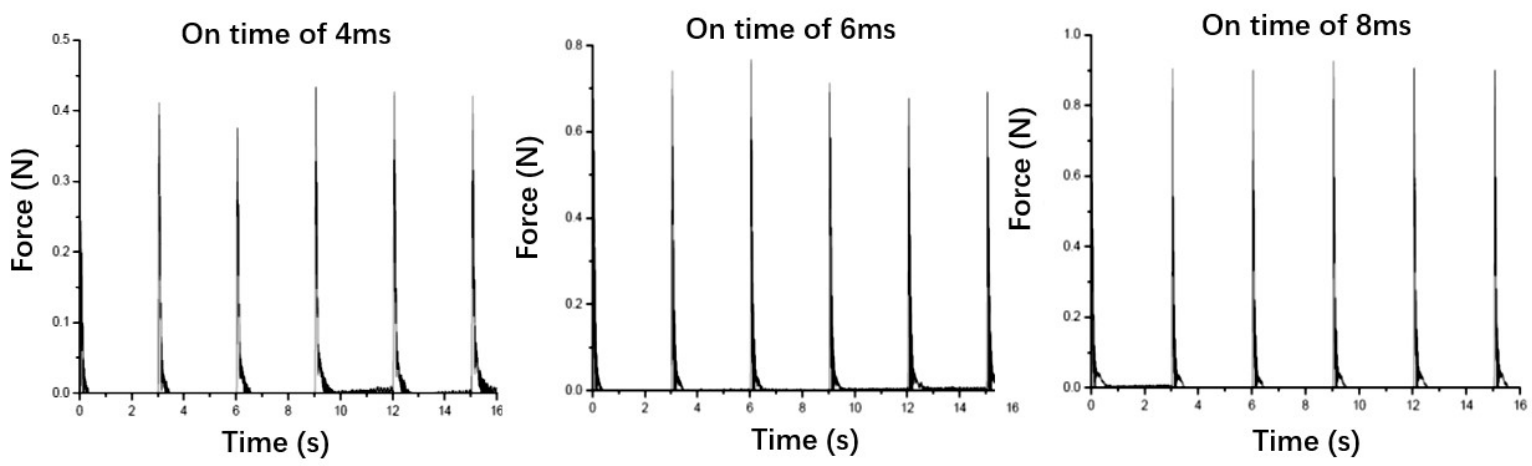

Figure 18. The short-impulse test data of thruster at different On time commands with $1 \mathrm{~m} / \mathrm{s}$ switch speed, $0.15 \mathrm{~mm}$ stroke with downstream throttle layout.

This result makes a good agreement with the firing test data. In the short-impulse test as shown in Figure 18, we can figure out thruster impulse characteristics at different On time commands. At On time of $4 \mathrm{~ms}$ command, the impulse repeatability is not good with $3 \delta$ over $60 \%$, because there is only $1 \mathrm{~ms}$ inadequate for stable spray and combustion. When On time rises, $3 \delta$ decreased to around $40 \%$ at $6 \mathrm{~ms}$ command and just $28 \%$ at $8 \mathrm{~ms}$ command. 
Increasing the switch speed and reducing the delay time of valve can be the only way to further improve dynamic response of bipropellant thrusters.

\section{Conclusions}

The transient spray atomization under impulse working conditions was numerically investigated, motivated by improving dynamic response of bipropellant thrusters. In particular, parametric studies were conducted to study the effects of high-speed solenoid valve characteristics on transient atomization of downstream injector.

As the solenoid valve switches to open, flowrate fluctuation immediately begins and then gradually stabilizes over time. When switch speed decreases to a certain extent, the fluctuation is strongly enhanced with negative influence on the downstream spray instabilities. Comparatively, NTO flows are more likely than MMH flows to produce such violent fluctuation. However, increasing the stroke can significantly reduce the fluctuation and improve the flow stability. Downstream throttle layout of orifice plate allows for greater transient velocity and benefits efficient atomization.

Both MMH spray and NTO spray can firstly reach a peak of droplets surface area under the strong flow fluctuation, and then MMH spray needs $3 \mathrm{~ms}$ for stabilization, while NTO spray requires $5 \mathrm{~ms}$ to be stable. Compared with switch speed and stroke, throttle layout plays more important role in influencing the spray development. After comparison, $1 \mathrm{~m} / \mathrm{s}$ switch speed, $0.15 \mathrm{~mm}$ valve stroke and downstream throttle layout can be a better combination for optimization. The following firing test data proved the simulation results that the atomization development time of $5 \mathrm{~ms}$ is required to obtain stable and repeatable impulse for bipropellant thruster.

\section{Acknowledgments}

This work is supported by National Natural Science Foundation of China (U1837211), the scholarship from China Scholarship Council (CSC) under the Gant CSC 201804980052, and Advanced Research Program in Equipment (513200602).

\section{Nomenclature}

None

\section{References}

[1] Gotzig, U. Schulte, G. Sowa, 1999, AIAA-99-2594.

[2] Fu, Q. F., Yang, L., J., 2015, Aerospace Science and Technology, 47, pp. 154-163.

[3] Hou, H., F., Wang, X., J., Vigor Y., 2014, AIAA, 0657.

[4] Arun, V., G., Shenbaga, V., N., Moorthi, A., M., 2015, Atomization and Sprays, 25(2), pp. 153-188.

[5] Yang, H. J., Chen, W., Lu, Z. Y., 2011, Proceedings of the CSEE, 31(24), pp. 52-57.

[6] Zhao L. M., Huai-Chao W. U, Zhao L., et al., 2018, IOP Conference Series: Materials Science and Engineering, 339(1), 012035, pp. 1-6.

[7] Rusche H., 2003, PhD Thesis, the University of London, UK.

[8] Berberovic E., 2010, PhD Thesis, Technische Universität, German.

[9] Zhang, Z., Shin D. T., 2020, International Journal of Spray and Combustion Dynamics, 12(2020):175682772093555.

[10] Sakman, A. T. Jog M A,Jeng S M, et al., 2000, AIAA Journal, 38(7), pp. 1214-1218.

[11] Lee, E. J., Oh, S. Y. Kim, H. Y., et al., 2010, Experimental Thermal and Fluid Science, 34(8), pp. 1475-1483. 